\title{
Effects of intra-arterial and intravenous transplantation of Induced Pluripotent Stem Cell-Derived Neural Progenitor Cells in ischemic stroke in rats
}

\author{
D.D. Namestnikova1', I.L. Gubskiy², E.A. Cherkashova1', I.A. Fedyunina3 ${ }^{3}$ D.I. Salikhova3 ${ }^{3}$
}

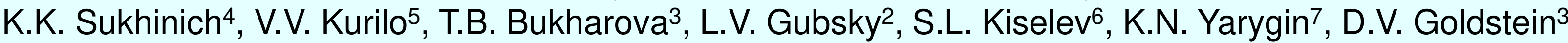

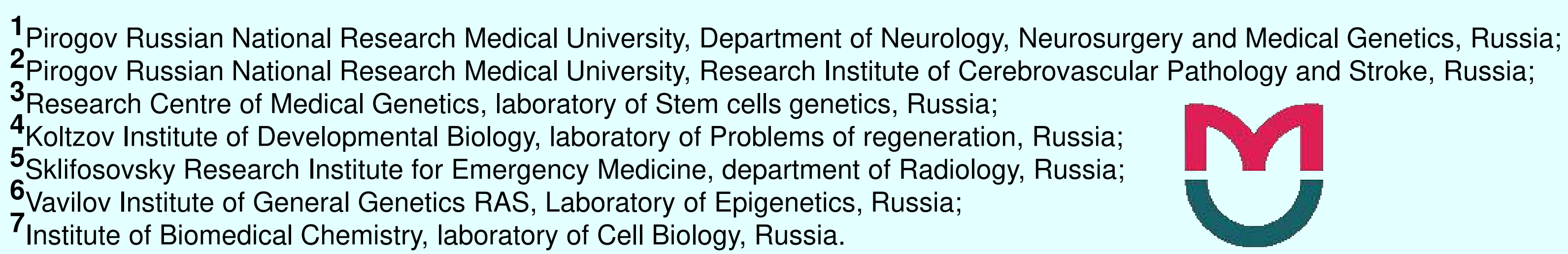

\section{Background and Aim}

Induced pluripotent stem cell-derived progenitor cells (iNPCs) can be genetically reprogrammed from a patient's own somatic cells and according to the recent pioneering research may be a promising therapeutic strategy for stroke therapy without ethical concerns and no risk of immune rejection. The aim of this study was to evaluate the therapeutic effects of iNPCs after their intra-arterial and intravenous transplantation in rats after MCAO.

Materials and Methods
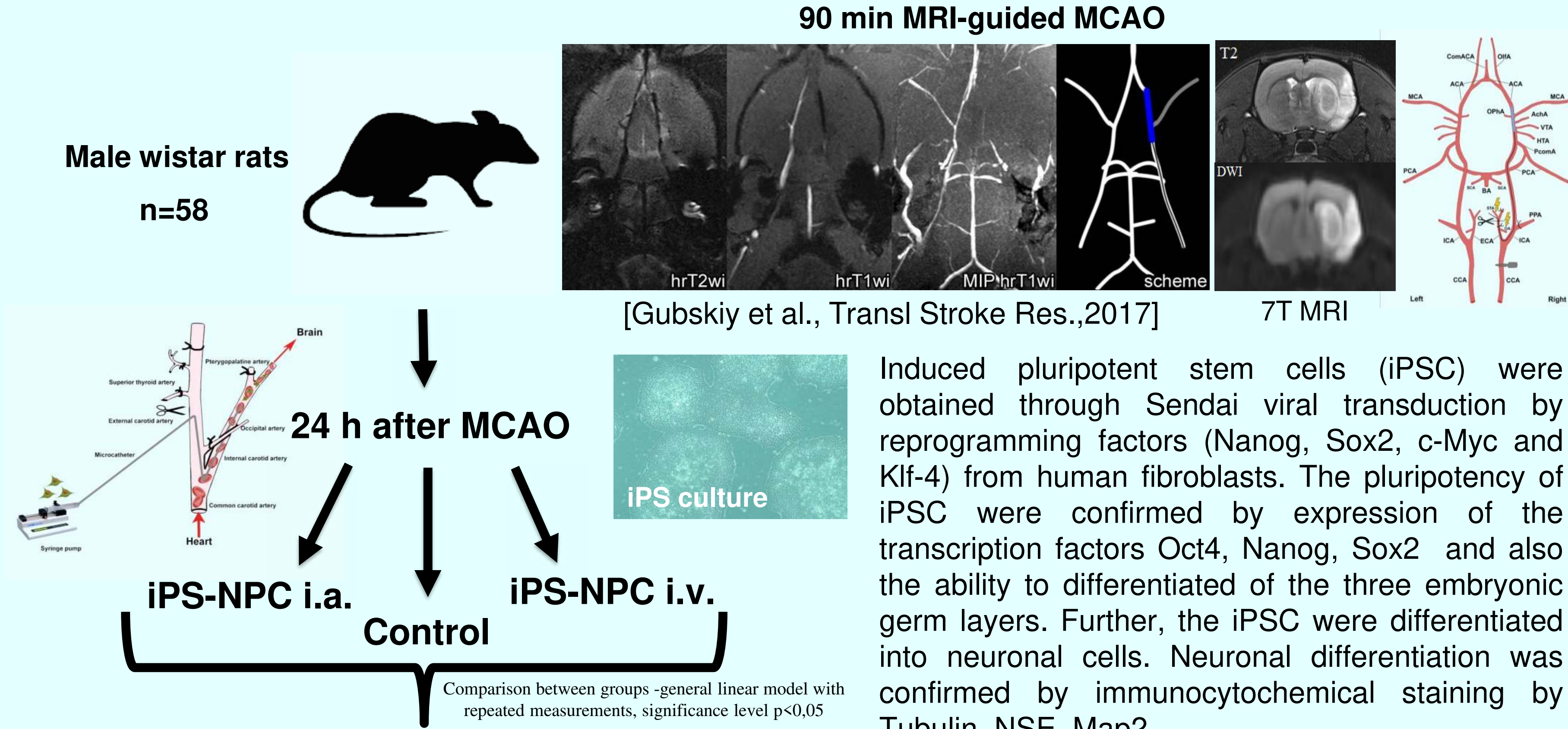

Modified neurological score scale

Stroke volume

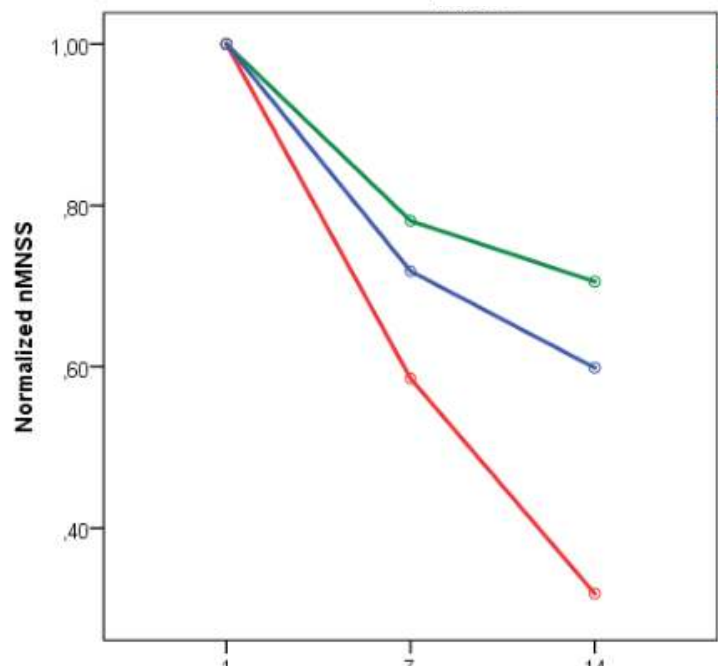

Survival

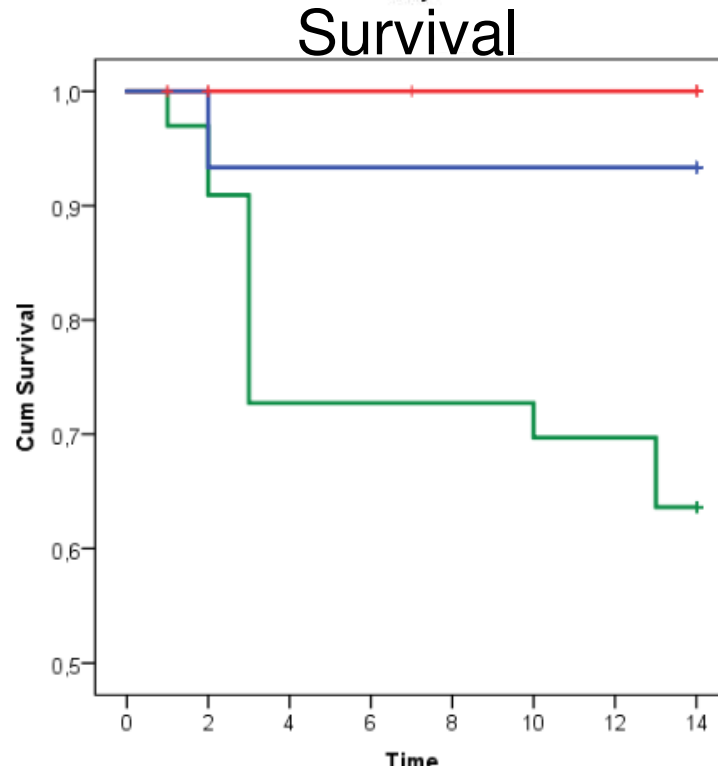

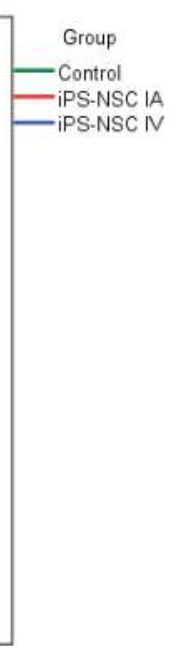

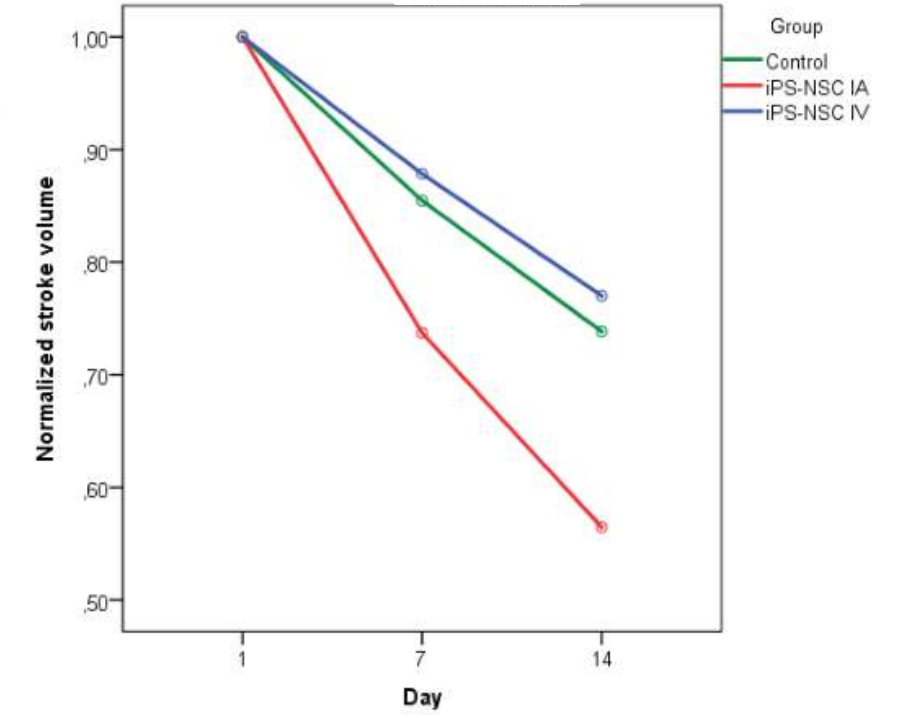

Fluorescent microscopy

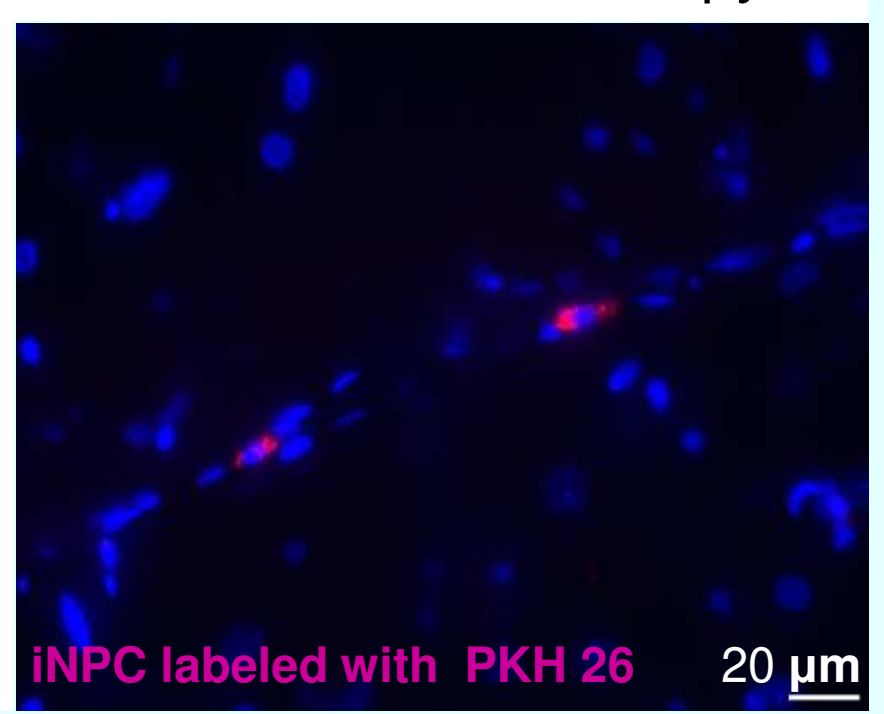

Induced pluripotent stem cells (iPSC) were obtained through Sendai viral transduction by reprogramming factors (Nanog, Sox2, c-Myc and KIf-4) from human fibroblasts. The pluripotency of iPSC were confirmed by expression of the transcription factors Oct4, Nanog, Sox2 and also the ability to differentiated of the three embryonic germ layers. Further, the iPSC were differentiated into neuronal cells. Neuronal differentiation was confirmed by immunocytochemical staining by Tubulin, NSE, Map2.
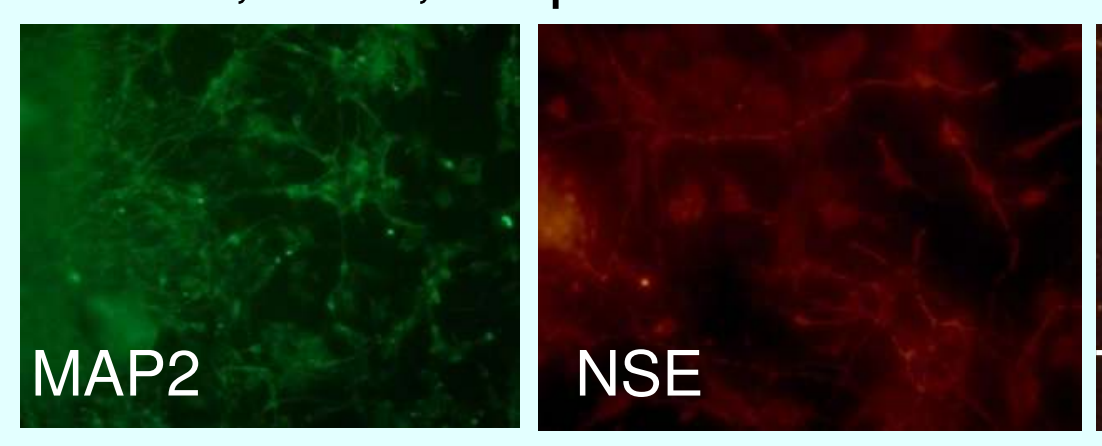

\section{Results}

Both I.V. and I.A. transplantation of iPS-NPC improved survival of animals. However, I.V. administration of iNPCs significantly reduced neurological deficit only 14d after MCAO, while IA administration of iNPCs reduced stroke volume and enhanced functional recovery already after $7 d$ from stroke onset. iPS-NPCs are capable to migrate through the blood-brain barrier after I.A. administration.

\section{Conclusions}

We have shown that systemic (IV and IA) transplantation of iNPCs are save and improves recovery after stroke. However, intra-arterial transplantation has more prominent therapeutic effects. 